
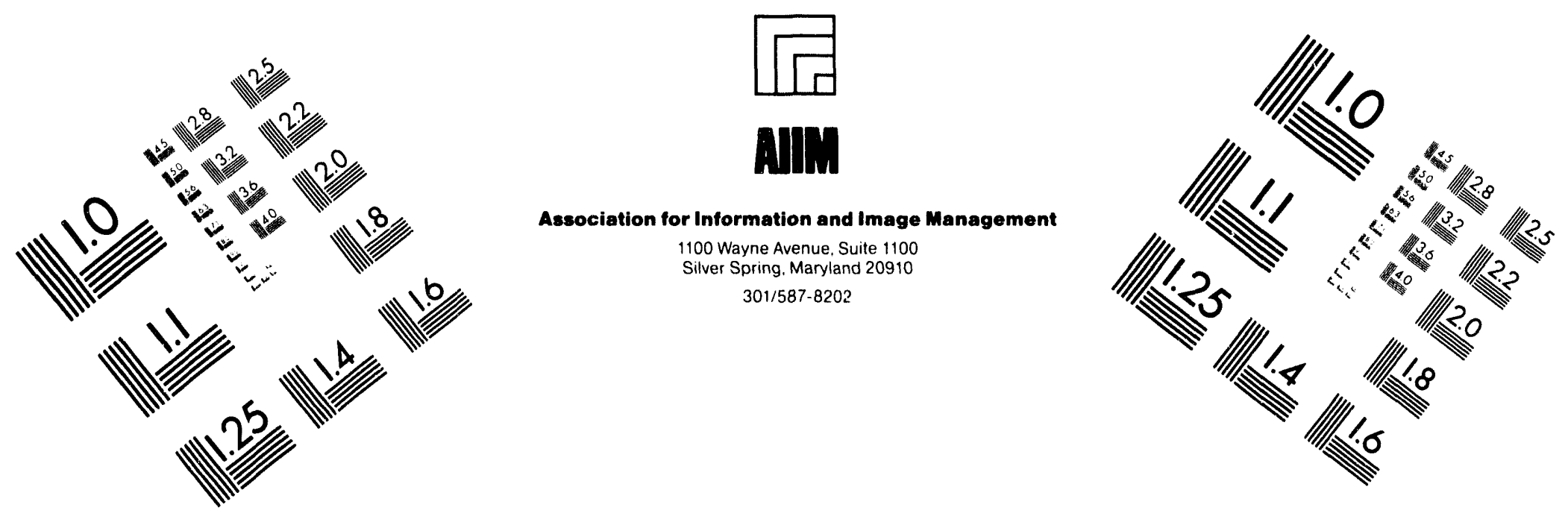

\title{
Centimeter
}

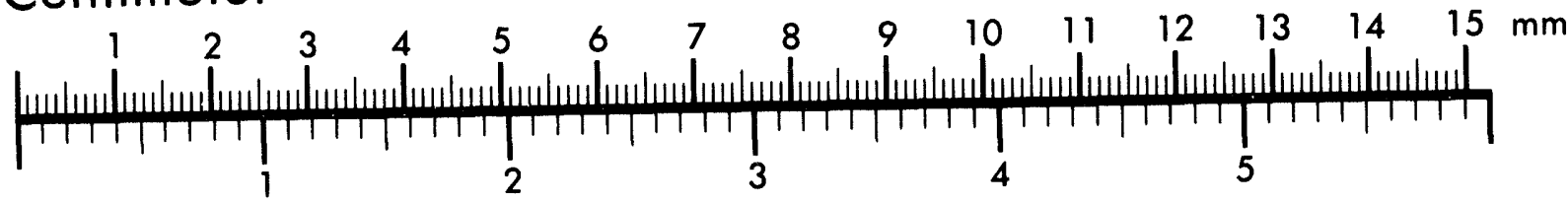

Inches
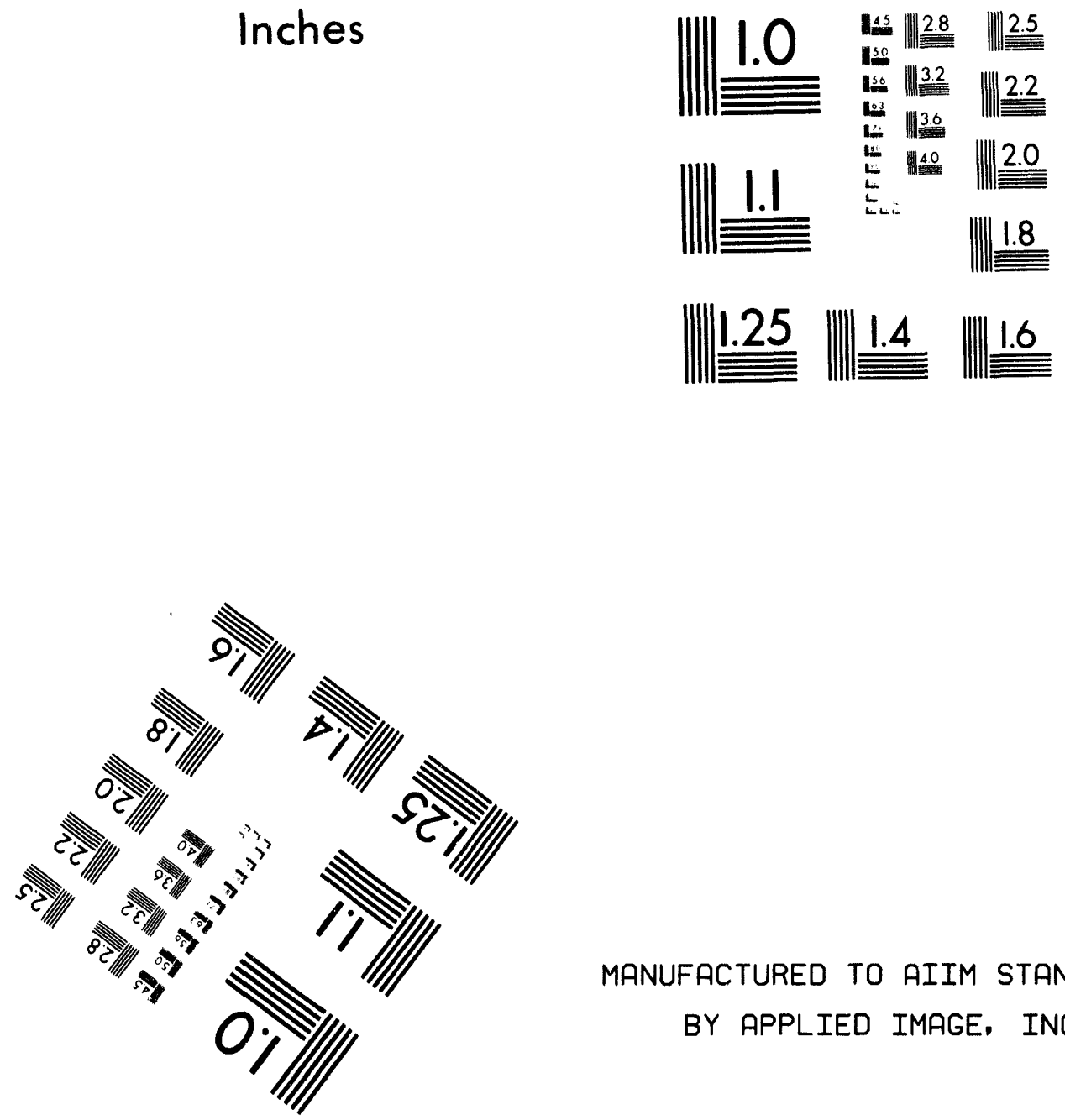

MANUFACTURED TO AIIM STANDARDS

BY APPLIED IMAGE, INC.

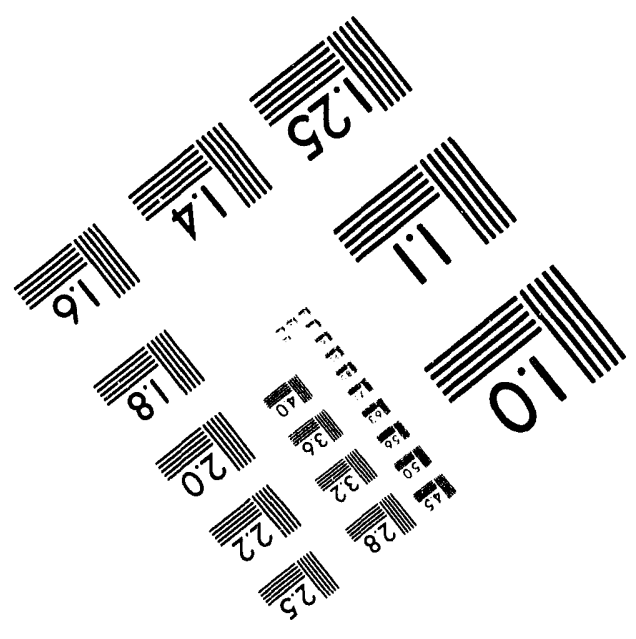



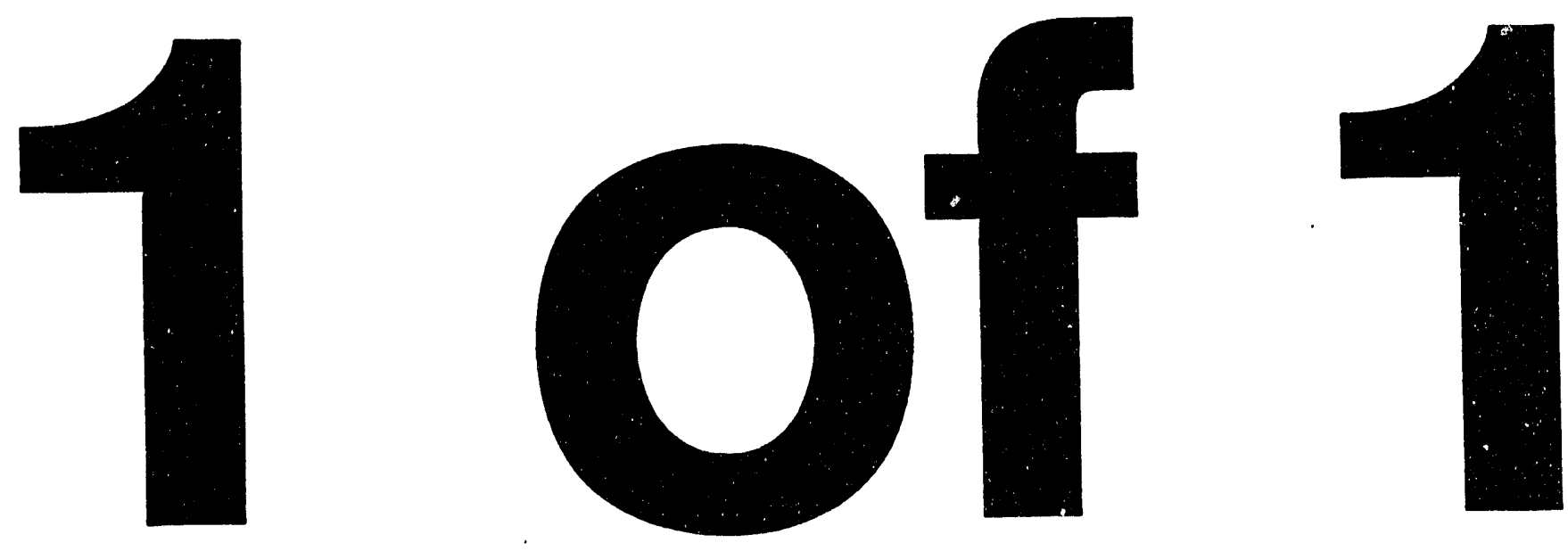
Computer Simulation Studies in Condensed Matter Physics, ed. by D. P. Landau, H.-B. Schüttler, and K. K. Mon, Springer-Verlag Publishing Co.

(Conference held at Athens, GA, February 21-25, 1994)

\section{STUDY OF FRUSTRATED SYSTEMS VIA DISSIPATIVE MAPS}

G. S. Canright and G. I. Watson

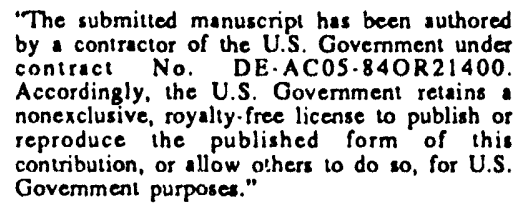

Governmenl purposes."

DEPARTMENT OF PHYSICS AND ASTRONOMY,
UNIVERSITY OF TENNESSEE, KNOXVILLE, TN

and

SOLID STATE DIVISION

OAK RIDGE NATIONAL LABORATORY

Managed by

MARTIN MARIETTA ENERGY SYSTEMS, INC.

Under

Contract No. DE-AC05-84OR21400

With the

U. S. DEPARTMENT OF ENERGY

OAK RIDGE, TENNESSEE

April 1994

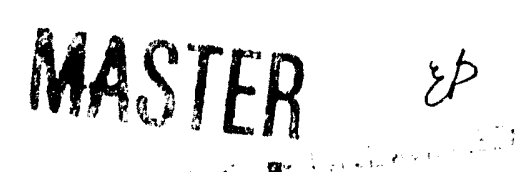




\title{
Study of Frustrated Systems via Dissipative Maps
}

\author{
G.S. Canright ${ }^{1,2}$ and G.I. Watson ${ }^{1,2,3}$ \\ ${ }^{1}$ Department of Physics and Astronomy, The University of Tennessee, \\ Knoxville, Tennessee 37996-1200 \\ 2Solid State Division, Oak Ridge National Laboratory, \\ Oak Ridge, Tennessee 37831 \\ ${ }^{3}$ Present address: Rutherford Appleton Laboratory \\ Chilton, Didcot, Oxon OX11, 0QX, U.K.
}

\begin{abstract}
We briefly describe a method for finding complex metastable states for frustrated classical systems, using a dissipative dynamical map. We show by example that this technique can give states which are very competitive in energy with the ground state, and with other, simpler states which are found by exhaustive search.
\end{abstract}

\section{Introduction: frustration}

We wish to consider "frustrated" problems in classical equilibrium statistical mechanics. Suppose we have an energy function

$$
F=\sum_{\text {pairs } i j} f_{|i-j|}\left(\theta_{i}, \theta_{j}\right)
$$

where the set $\left\{\theta_{i}\right\}$ represents the degrees of freedom of the problem. We call this function (and the system it represents) frustrated if it is impossible to choose a set of coordinates $\left\{\theta_{i}\right\}$ such that every term in $F$ is simultaneously minimized.

Since the equilibrium problem is precisely to minimize $F$, then clearly when $F$ is frustrated the equilibrium problem is in general nontrivial. Let us now further restrict our consideration to problems in which the frustration arises without any disorder in $F$. Even in this restricted class of problems, it is in general very difficult to deduce the absolute minimum of $F$. Furthermore, as $F$ becomes increasingly frustrated, the importance of the absolute minimum ("ground state") diminishes relative to that of other local minima (metastable states), due to the tendency for large activation barriers to appear between minima ( $F$ becomes "crumpled"). Hence we broaden the problem, in the case of frustration, to one of finding a representative set of low-energy ("cornpetitive") minima-a set which includes but is not limited to the ground state.

One interesting technique which has been applied to such problems involves writing the minimization problem as a mapping [1]. To pursue this idea we 
restrict ourselves further to one-dimensional problems. Then the minimization problem for (1) may be written as an infinite set of coupled equations

$$
\frac{\partial}{\partial \theta_{i}} F\left(\ldots \theta_{i-2}, \theta_{i-1}, \theta_{i}, \theta_{i+1}, \theta_{i+2}, \ldots\right)=0 \text {. }
$$

Here the coordinates are listed in 1D order. Assuming the interaction in $F$ extends to the $r$ th neighbor, there are $2 r+1$ coordinates in each of (2). The $i$ th equation may then be rewritten as an equation for $\theta_{i+r}$ in terms of ti:e $2 r$ previous $\theta$ 's. In this light, knowledge of those previous $2 r$ values for $\theta$ will determine $\theta_{i+r}$, from (2). Thus we obtain the $2 r$-dimensional map

$$
\left(\begin{array}{c}
\theta_{i+r-1} \\
\theta_{i+r-2} \\
\vdots \\
\theta_{i-r}
\end{array}\right) \Longrightarrow\left(\begin{array}{c}
\theta_{i+r} \\
\theta_{i+r-1} \\
\vdots \\
\theta_{i-r+1}
\end{array}\right) .
$$

Trajectories of (3) then correspond to extrema of $F$. for whatever $2 r$ boundary conditions are used to start the iteration. Such maps have two important features [1]: (i) they are volume-preserving; (ii) stable trajectories of the mapping correspond to unstable extrema of $F$, and vice versa. This latter feature makes it difficult to study complex minima of $F$ [say, those represented by long-period or chaotic trajectories of (3)], although simpler structures given by fixed points of (3) can be located accurately.

\section{Dissipative maps}

We could solve problem (ii) above if we could replace the volume-preserving mapping (3) with a dissipative map--one whose orbits converge to attractors in the phase space - and if we could arrange that these attractors be low-energy minima (if not all the minima) of $F$. Such a mapping could then act as a 'magic bullet' which would efficiently locate those minima we want to study. This would include minima of arbitrary complexity. For example, with such a 'magic bullet' we could find disordered minima of $F$ from chaotic attractors of the dissipative map, evaluate their energetics accurately, and otherwise characterize them in terms of correlations, etc.

We have discovered an approximate magic bullet, and applied it to a frustrated 1D problem of some physical interest [2]. In this problem $F$ is of the form

$$
F=\sum_{n=1}^{\infty} \sum_{n^{\prime}=n+1}^{n+r} f_{\left|n-n^{\prime}\right|}\left(\left|\theta_{n}-\theta_{n^{\prime}}\right|\right)
$$

where $r$ is in principle infinite, but effectively finite (see Section 3). For this case our method may be described as follows. We believe [2] that there are regular 
solutions to (2) [with $F$ as in (4)] of the form $\theta_{j}=j \phi$. We then consider a somewhat weaker condition: let $\left.\bar{F}(\phi) \equiv F\right|_{\theta,=j \phi}$, and assume that

$$
\frac{\partial \bar{F}(\phi)}{\partial \phi}=0
$$

that is, that there are uniform configurations which are stable against variation of the lattice constant. This is equivalent to

$$
\sum_{\ell=1}^{r} \ell f_{\ell}^{\prime}(\ell \phi)=0
$$

where $f_{n}^{\prime}(x) \equiv \partial f_{n}(x) / \partial x$.

Now we want a dissipative map which agrees with (6). We imagine (as with the volume-preserving map) "growing" the lattice from the left, but letting the choice $\theta_{j}^{*}$ of each new $\theta_{j}$ be a one-dimensional minimization problem:

$$
G\left(\theta_{j}^{*}\right)=\min _{\theta,} G\left(\theta_{j}\right) \text { with } G\left(\theta_{j}\right)=\sum_{n=j-1}^{n=j-r} g_{|j-n|}\left(\left|\theta_{j}-\theta_{n}\right|\right) .
$$

Since $\theta_{j}$ is then determined by the $r$ previous values, this gives an $r$-dimensional map similar to (3). However, we choose a fictitious two-body potential $g$ to replace the true potential $f$. It is then easily shown [2] that fixed points of the resulting map will correspond exactly to solutions to (6) (ie, to a uniform configuration stable with respect to variation in the uniform spacing $\phi$ ) if we set

$$
g_{\ell}(x)=\ell f_{\ell}(x) .
$$

In other words, we use knowledge about simple minima of $F$ (eg, that uniform minima exist) to determine the fictitious potential $G$. The latter then gives rise to a dissipative map (7). Some of the attractors of this map will be (exactly) the 'simple' minima of $F$. However, we suppose that the mapping built from $G$ will have other attractors of more complex structure, and that these other attractors will be close to low-energy minima of $F$. (The nearby exact minima may then be found by standard, purely local relaxation schemes.) This supposition has already been well supported by [2]; below we offer a further test of the idea.

\section{Results: complex structures at low energy}

In this section we wish to compare metastable states, found using the dissipative map (DM) described above, with other (small-period) metastable states found by an "exhaustive search" (ES) procedure. We will see (Fig. 1) that the DM can guide us to states of high complexity (including disordered states) which 


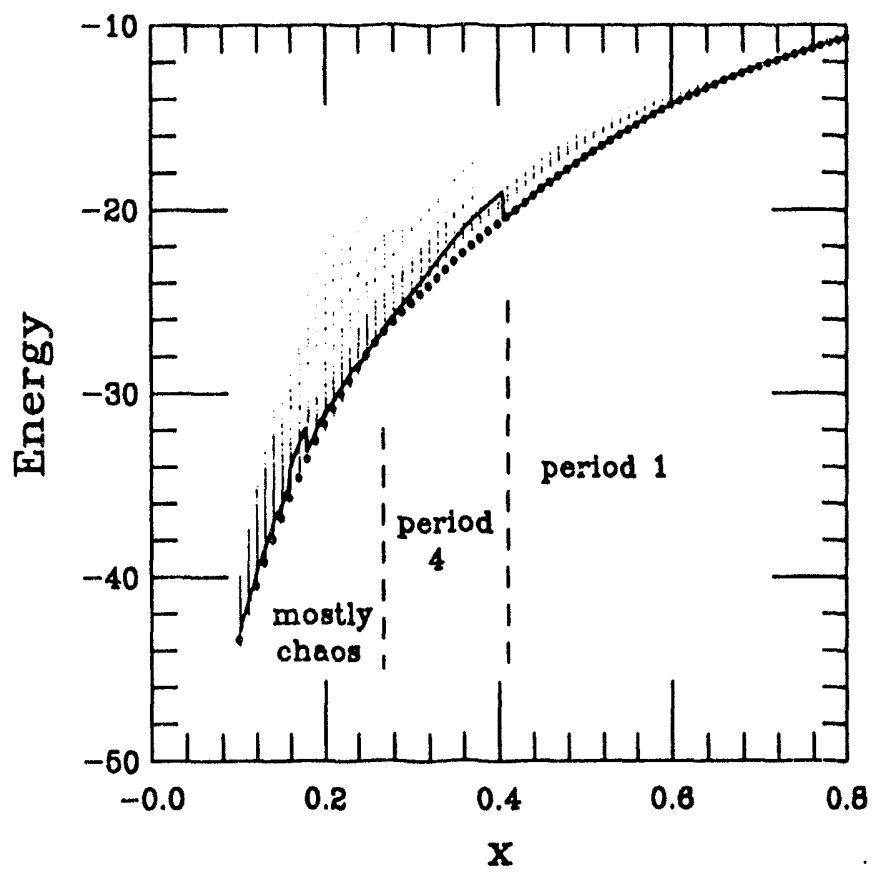

Figure 1. Energies for metastabie states of a frustrated "spin chain", as a function of $x(\approx$ the inverse range of the antiferromagnetic interaction). Small dots: periodic minima found by "exhaustive search" (see text), for periods $\leq 8$. Large dots: typical period-1 minima. Solid line: minima found using a dissipative map (followed by local relaxation). Structures of these minima are labelled in the figure. Note that, using the DM, we have found structures of great complexity (period $\rightarrow \infty$ ) which are nevertheless very low in energy.

(a) could never be found by any exhaustive search procedure, and yet (b) are highly competitive in energy with those states which we can find by exhaustive search.

The problem we consider is that of Ref. [2]. Our two-body interaction $f$ (representing repulsion between uniform layers of flux lines in a layered superconductor) is then $f_{n}(\alpha)=$ (const) $\log \left(1+e^{-4 n x}-2 e^{-2 n x} \cos 2 \pi \alpha\right)$. Here $\alpha \equiv\left|\theta_{i}-\theta_{j}\right|, n=|i-j|$, and $0 \leq \theta_{i}<1$. The parameter $x$ is derived from the average flux line density. Small $x$ makes $f$ more long-ranged, and so (since $f$ is uniformly repulsive) makes $F$ more frustrated. For simplicity we may think of our 1D chain of layers as a classical XY spin chain, with the spin couplings long-ranged (range $\propto 1 / x$ ) and uniformly antiferromagnetic.

Our ES procedure is similar to one described in Peyrard and Aubry [3]. To 
consider metastable states of period $p$, we study chains of length $K>1 / x$ (with $K$ a multiple of $p$ ) and periodic boundary conditions. The configuration space for $K$ spins can then be divided into sectors defined by the relative ordering of the spins in the unit interval. It is plausible that, for $K$ larger than the range of interaction, each such sector contains (at most) a single minimum of $F$. We then seek that minimum by choosing a starting point in each sector (each spin getting one of the values $\ell / K, \ell=0, \ldots, K-1$ ) and relaxing (using standard routines) to the nearest local minimum.

A comparison of the ES energies with those found using the DM is given in Fig. 1. We see that, at small $x$, the DM allows us to find disordered (chaotic) minima of the energy (as well as large-period structures) [2] which are highly competitive with the simpler structures found by exhaustive search. Our method of finding these structures, using the DM, is unique (as far as we know) in this regard: that is, we know of no other method for finding these structur's which is efficient enough to be practicable.

We are confident that this approach may be usefully applied to other frustrated ! D problems [4]. An extension to 2D (or higher) is of great interest. This may be possible in a restricted sense by using periodic boundary conditions in the other dimension(s); however this kills the possibility of observing structures which have an arbitrary period in either dimension. A more interesting problem (which we do not yet know how to solve) is to write an iterated mapping to represent the energetics for $\mathrm{D}>1$, if open $\mathrm{BC}$ are used in all dimensions.

\section{Acknowledgments}

We would like to thank David Phillippi for valuable contributions in the early stages of this research, and Ofer Biham for suggesting the exhaustive search. We also thank Ofer Biham, Ron Fox, and Andy Zangwill for helpful discussions and references. This work was supported by the University of Tennessee and the Division of Materials Science, U.S. Department of Energy under Contract No. DE-AC05-84OR21400 with Martin Marietta Energy Systems. GSC was supported in part by the NSF under grant \# DMR-9101542.

\section{References}

1. See, for example, Per Bak, Phys. Rev. Lett. 46, 791 (1981); Rahul Pandit and Michael Wortis, Phys. Rev. B 25, 3226 (1982); and Ref. [3].

2. G.I. Watson and G.S. Canright, Phys. Rev. B 48, 15950 (1993).

3. M. Peyrard and S. Aubry, J. Phys. C 16, 1593 (1983).

4. We have explored limited applications of the method to the ANNNI problem [Julia Yeomans, Solid State Physics 41, 151 (1988)] and to the long-range oscillatory potentials of metallic close-packed polytypes [A. Blandin, J. Friedel, and G. Saada, J. Phys. (Paris) Colloq. 27, C3-128 (1966)]. 

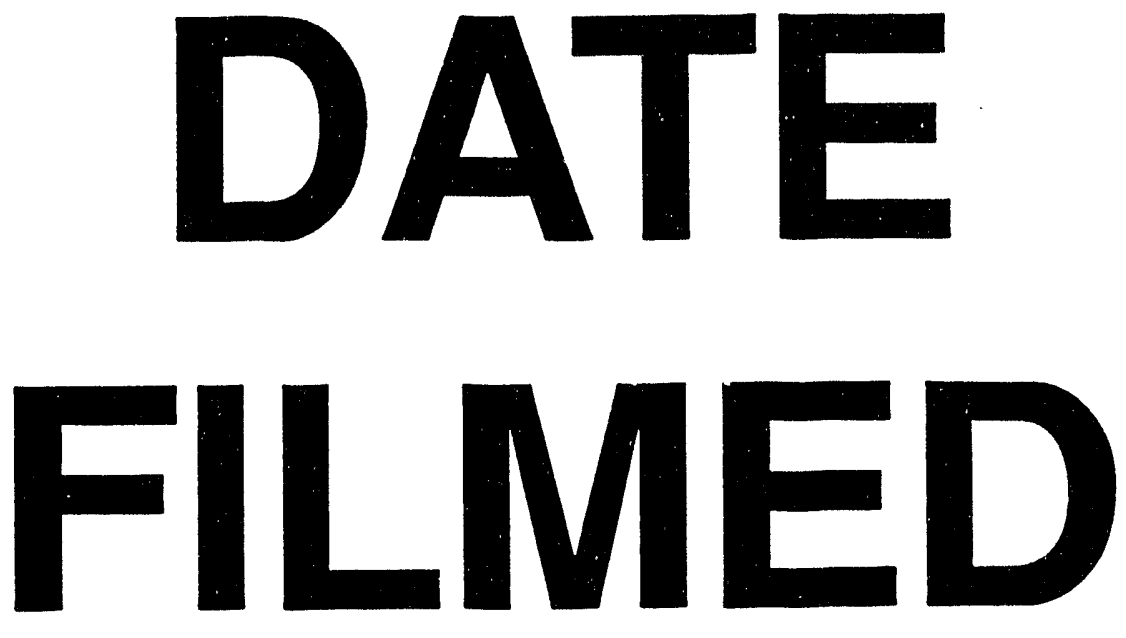

$10 / 20 / 94$
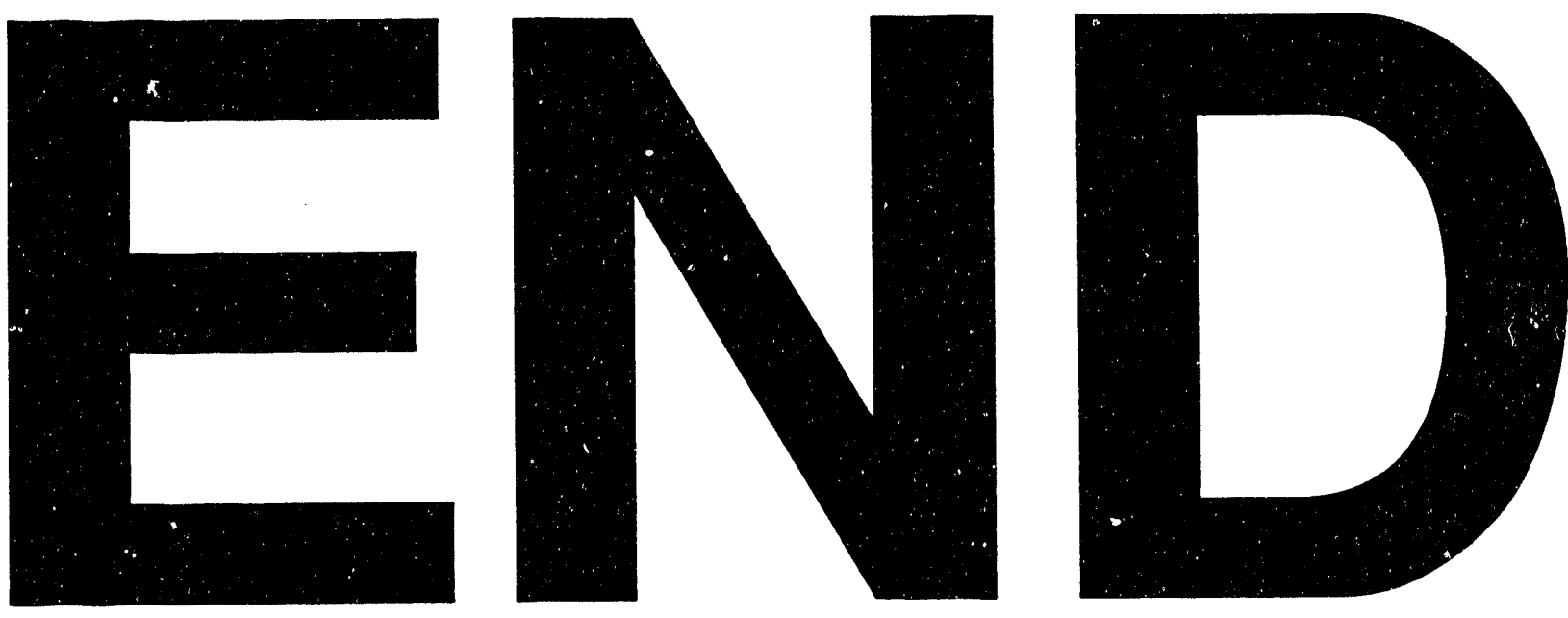
